\title{
Bicomplex Numbers: Further Contributions to a Fibonacci and Fibonacci - Lucas Matrices Oriented Approach
}

\author{
Faik BABADAĞ $\breve{G}^{*}$ \\ Kırlkkale University, Faculty of Arts and Sciences, Department of Mathematics, Kırlkkale, Turkey \\ (ORCID: 0000-0001-9098-838X)
}

\begin{abstract}
In this study, by using Fibonacci $Q$-matrix and Lucas $Q^{\prime}$-matrix we define bicomplex Fibonacci $Q$-matrix and bicomplex Lucas $Q^{\prime}$-matrix. After that using this matrix representation, we give some identities.

Keywords: Bicomplex number, Fibonacci $\mathcal{Q}$-matrix, Fibonacci and Lucas numbers.

\section{Bikompleks Sayllar: Fibonacci ve Fibonacci-Lucas Matrislerine Yönelik Yaklaşımına İlave Katkılar}

$\ddot{O} \mathbf{z}$

$\mathrm{Bu}$ çalışmada, Fibonacci $\mathcal{Q}$-matrisi ve Lucas $\mathcal{Q}^{\prime}$-matrisi kullanarak bikompleks Fibonacci $\mathcal{Q}$-matris ve bikompleks Lucas $Q^{\prime}$-matrisi tanımladık. Daha sonra bu matris sunumunu kullanarak bazı özdeşlikler verdik.

Anahtar kelimeler: Bikompleks sayı, Fibonacci $\mathcal{Q}$-matris, Fibonacci ve Lucas sayılar.

\section{Introduction}

A bicomplex number is described by

$\mathcal{B}=a+i b+j c+i j d$,

where the imaginary units $i, j$ and $i j$ are governed by the rules: $i^{2}=j^{2}=-1,(i j)^{2}=(j i)^{2}=+1$. For two bicomplex numbers $\mathcal{B}=a+i b+j c+i j d$ and $\mathcal{B}^{\prime}=a^{\prime}+i b^{\prime}+j c^{\prime}+i j d^{\prime}$, the addition, subtraction and multiplication of these numbers are given by

$\mathcal{B} \mp \mathcal{B}^{\prime}=\left(a \mp a^{\prime}\right)+i\left(b \mp b^{\prime}\right)+j\left(c \mp c^{\prime}\right)+i j\left(d \mp d^{\prime}\right)$

and

$$
\begin{aligned}
\mathcal{B} \times \mathcal{B}^{\prime}= & (a+i b+j c+i j d) \times\left(a^{\prime}+i b^{\prime}+j c^{\prime}+i j d^{\prime}\right) \\
= & \left(a a^{\prime}-b b^{\prime}-c c^{\prime}+d d^{\prime}\right)+i\left(a b^{\prime}+b a^{\prime}-c d^{\prime}-d c^{\prime}\right) \\
& +j\left(a c^{\prime}+c a^{\prime}-b d^{\prime}-d b^{\prime}\right)+i j\left(a d^{\prime}+d a^{\prime}+c b^{\prime}+b c^{\prime}\right),
\end{aligned}
$$

respectively. The conjugates of the bicomplex number $\mathcal{B}$ are denoted by $\mathcal{B}^{i}, \mathcal{B}^{j}$ and $\mathcal{B}^{i j}$. In that case, there are different conjugations as follows, [1]: 


$$
\begin{aligned}
& \mathcal{B}^{i}=a-i b+j c-i j d, \\
& \mathcal{B}^{j}=a+i b-j c-i j d, \\
& \mathcal{B}^{i j}=a-i b-j c+i j d .
\end{aligned}
$$

The Fibonacci and Lucas sequence are presented for all integers $n$ by the second order recurrence relation $f_{n+2}=f_{n+1}+f_{n}$ and initial conditions $f_{1}=f_{2}=1$ and $l_{n+2}=l_{n+1}+l_{n}$ but initial conditions $l_{1}=1, l_{2}=3$. Different applications of Fibonacci and Lucas numbers have been in almost all fields of science, $[2,11]$.

The Fibonacci $\mathcal{Q}$-matrix and Lucas $\mathcal{Q}^{\prime}$-matrix are presented as, [12, 15];

$\mathcal{Q}=\left[\begin{array}{ll}1 & 1 \\ 1 & 0\end{array}\right] \quad$ and $\quad \mathcal{Q}^{\prime}=\left[\begin{array}{ll}3 & 1 \\ 1 & 2\end{array}\right]$

The $n^{\text {th }}$ power of the $Q$-matrix and $\mathcal{Q}^{\prime}$-matrix are

$\mathcal{Q}^{n}=Q_{\mathrm{n}}=\left[\begin{array}{cc}f_{n+1} & f_{n} \\ f_{n} & f_{n-1}\end{array}\right]$ and $\left(\mathcal{Q}^{\prime}\right)^{n}=\mathcal{Q}_{n}^{\prime}=\left[\begin{array}{cc}\ell_{n+1} & \ell_{n} \\ \ell_{n} & \ell_{n-1}\end{array}\right]$.

Furthermore, it is clearly expressed as $f_{n-1} f_{n+1}-f_{n}^{2}=(-1)^{\mathrm{n}}$ and

$\mathcal{Q}^{n+1} \mathcal{Q}^{n}=\mathcal{Q}^{2 n+1}=\mathcal{Q}_{\mathrm{n}+1} \mathcal{Q}_{\mathrm{n}}=\mathcal{Q}_{2 \mathrm{n}+1}$

In this present paper, by combining bicomplex numbers and Fibonacci, Lucas numbers we define bicomplex Fibonacci $Q$-matrix and bicomplex Lucas $Q^{\prime}$-matrix. We define some properties.

The bicomplex Fibonacci and bicomplex Lucas number are given respectively by

$\mathcal{B} f_{\mathrm{n}}=f_{n}+i f_{n+1} i+j f_{n+2}+i j f_{n+3}$ and $\mathcal{B} \ell_{\mathrm{n}}=\ell_{n}+i \ell_{n+1}+j \ell_{n+2}+i j \ell_{n+3}$,

where $f_{n}$ and $\ell_{n}$ are the $n^{\text {th }}$ Fibonacci numbers, Lucas numbers and $i^{2}=j^{2}=-1,(i j)^{2}=+1$.

If we start from $n \geq 0$, the bicomplex Fibonacci and bicomplex Lucas number are given as;

$\mathcal{B} f_{0}=i+j+2 i j ; \mathcal{B} f_{1}=1+i+2 j+3 i j ; \mathcal{B} f_{2}=1+2 i+3 j+5 i j$

and

$\mathcal{B} \ell_{0}=2+i+3 j+4 i j ; \quad \mathcal{B} \ell_{1}=1+3 i+4 j+7 i j ; \mathcal{B} \ell_{2}=3+4 i+7 j+11 i j$.

\section{Bicomplex Fibonacci $\mathcal{Q}$-Matrix and Bicomplex Lucas $\mathcal{Q}^{\prime}$-Matrix}

For $n \geq 0$, the $n^{\text {th }}$ bicomplex Fibonacci $\mathcal{Q}_{n}$-matrix $\mathcal{B}_{\mathrm{n}}$ and the $n^{\text {th }}$ bicomplex Lucas $\mathcal{Q}_{n}^{\prime}$-matrix $\mathcal{B}_{\mathrm{n}}^{\prime}$ are defined as

$\mathcal{B}_{\mathrm{n}}=Q_{n}+i Q_{n+1}+j Q_{n+2}+i j Q_{n+3}$ and $\mathcal{B}_{\mathrm{n}}^{\prime}=Q_{n}^{\prime}+i Q_{n+1}^{\prime}+j Q_{n+2}^{\prime}+i j Q_{n+3}^{\prime}$,

where $i, j$ and $i j$ are arbitrary units which satisfy the relations; $i^{2}=j^{2}=-1,(i j)^{2}=+1$.

Now we will give some identities on bicomplex Fibonacci $\mathcal{Q}$-matrix.

Identities 1. For $m, n \geq 0$,

$\mathcal{B}_{n}-i \mathcal{B}_{n+1}-j \mathcal{B}_{n+2}+i j \mathcal{B}_{n+3}=-Q_{n}^{\prime}\left[\begin{array}{ll}7 & 4 \\ 4 & 3\end{array}\right]$, 
$\mathcal{B}_{n} \times \mathcal{B}_{n}^{i j}+\mathcal{B}_{n-1} \times \mathcal{B}_{n-1}^{i j}=5 \mathcal{Q}_{2 n}\left[\begin{array}{ll}2 & 1 \\ 1 & 1\end{array}\right]$

$\mathcal{B}_{\mathrm{n}} \times \mathcal{B}_{\mathrm{m}}=(1-2 i)\left(Q_{m+n+3}^{\prime}-2 j \mathcal{Q}_{m+n+3}\right)$,

where $\mathcal{B}_{n}^{i j}$ is the conjugation with respect to the imaginary unit $i j$.

Proof . We will give the proof of identity $\mathcal{B}_{n}-i \mathcal{B}_{n+1}-j \mathcal{B}_{n+2}+i j \mathcal{B}_{n+3}=3 \mathcal{Q}_{n}^{\prime}\left[\begin{array}{ll}7 & 4 \\ 4 & 3\end{array}\right]$. Using equality (5), we have

$$
\begin{aligned}
= & Q_{n}+i Q_{n+1}+j Q_{n+2}+i j Q_{n+3}-i\left(Q_{n+1}+i Q_{n+2}+j Q_{n+3}+i j Q_{n+4}\right) \\
& -j\left(Q_{n+2}+i Q_{n+3}+j Q_{n+4}+i j Q_{n+5}\right)+i j\left(Q_{n+3}+i Q_{n+4}+j Q_{n+5}+i j Q_{n+6}\right) \\
= & Q_{n}+Q_{n+2}+Q_{n+4}+Q_{n+6},
\end{aligned}
$$

from the equality (2) and the identities $f_{n-1}+f_{n+1}=l_{n}$ and $f_{n+4}+f_{n}=3 f_{n+2}$ in $[3,5]$, we can write as

$$
\begin{aligned}
& =\left[\begin{array}{cc}
f_{n+1} & f_{n} \\
f_{n} & f_{n-1}
\end{array}\right]+\left[\begin{array}{ll}
f_{n+3} & f_{n+2} \\
f_{n+2} & f_{n+1}
\end{array}\right]+\left[\begin{array}{cc}
f_{n+5} & f_{n+4} \\
f_{n+4} & f_{n+3}
\end{array}\right]+\left[\begin{array}{ll}
f_{n+7} & f_{n+6} \\
f_{n+6} & f_{n+5}
\end{array}\right] \\
& =3\left[\begin{array}{ll}
l_{n+4} & l_{n+3} \\
l_{n+3} & l_{n+2}
\end{array}\right] \\
& =3\left[\begin{array}{cc}
l_{n+1} & l_{n} \\
l_{n} & l_{n-1}
\end{array}\right]\left[\begin{array}{ll}
l_{4} & l_{3} \\
l_{3} & l_{2}
\end{array}\right],
\end{aligned}
$$

from the equality (2), we obtain

$$
=3 Q_{n}^{\prime} Q_{3}^{\prime}=3 Q_{n}^{\prime}\left[\begin{array}{ll}
7 & 4 \\
4 & 3
\end{array}\right] .
$$

Now we will prove the identity

$$
\mathcal{B}_{n} \times \mathcal{B}_{n}^{i j}+\mathcal{B}_{n-1} \times \mathcal{B}_{n-1}^{i j}=5 \mathcal{Q}_{2 n}\left[\begin{array}{ll}
2 & 1 \\
1 & 1
\end{array}\right] .
$$

By using the equalities (1), (2) and (5), we get

$$
=Q_{n-1}^{2}+2 Q_{n}^{2}-2 Q_{n+2}^{2}-Q_{n+3}^{2},
$$

if we rewrite the equality (6) from equality (3),

$$
\begin{aligned}
& =Q_{2 n-2}+2 Q_{2 n}-2 Q_{2 n+4}-Q_{2 n+6} \\
& =\left[\begin{array}{ll}
f_{2 n-1} & f_{2 n-2} \\
f_{2 n-2} & f_{2 n-3}
\end{array}\right]+2\left[\begin{array}{cc}
f_{2 n+1} & f_{2 n} \\
f_{2 n} & f_{2 n-1}
\end{array}\right]-2\left[\begin{array}{cc}
f_{2 n+5} & f_{2 n+4} \\
f 2_{n+4} & f_{2 n+3}
\end{array}\right]-\left[\begin{array}{ll}
f_{2 n+7} & f_{2 n+6} \\
f_{2 n+6} & f_{2 n+5}
\end{array}\right]
\end{aligned}
$$

From equality (7), we obtain

$=\left[\begin{array}{cc}l_{2 n} & l_{2 n-1} \\ l_{2 n-1} & l_{2 n-2}\end{array}\right]-\left[\begin{array}{ll}l_{2 n+3} & l_{2 n+2} \\ l_{2 n+2} & l_{2 n+1}\end{array}\right]-\left[\begin{array}{ll}l_{2 n+6} & l_{2 n+5} \\ l_{2 n+5} & l_{2 n+4}\end{array}\right]$ 


$$
\begin{aligned}
& =5\left[\begin{array}{ll}
f_{2 n+3} & f_{2 n+2} \\
f_{2 n+2} & f_{2 n+1}
\end{array}\right] \\
& =5\left[\begin{array}{cc}
f_{2 n+1} & f_{2 n} \\
f_{2 n} & f_{2 n-1}
\end{array}\right]\left[\begin{array}{ll}
f_{3} & f_{2} \\
f_{2} & f_{1}
\end{array}\right] \\
& =5 Q_{2 n}\left[\begin{array}{ll}
2 & 1 \\
1 & 1
\end{array}\right] .
\end{aligned}
$$

Lastly, considering equality (5), we have

$$
\begin{aligned}
\mathcal{B}_{\mathrm{n}} \times \mathcal{B}_{\mathrm{m}}= & \left(\mathcal{Q}_{n} \mathcal{Q}_{m}-\mathcal{Q}_{n+1} \mathcal{Q}_{m+1}-\mathcal{Q}_{n+2} \mathcal{Q}_{m+2}+\mathcal{Q}_{n+3} \mathcal{Q}_{m+3}\right) \\
& +i\left(\mathcal{Q}_{n} \mathcal{Q}_{m+1}+\mathcal{Q}_{n+1} \mathcal{Q}_{m}-\mathcal{Q}_{n+2} \mathcal{Q}_{m+3}-\mathcal{Q}_{n+3} \mathcal{Q}_{m+2}\right) \\
& +j\left(\mathcal{Q}_{n} \mathcal{Q}_{m+2}+\mathcal{Q}_{n+2} \mathcal{Q}_{m}-\mathcal{Q}_{n+1} \mathcal{Q}_{m+3}-\mathcal{Q}_{n+3} \mathcal{Q}_{m+1}\right) \\
& +i j\left(\mathcal{Q}_{n} \mathcal{Q}_{m+3}+\mathcal{Q}_{n+3} \mathcal{Q}_{m}+\mathcal{Q}_{n+2} \mathcal{Q}_{m+1}+\mathcal{Q}_{n+1} \mathcal{Q}_{m+2}\right) \\
= & Q_{m+n}\left(Q_{0}-\mathcal{Q}_{2}-\mathcal{Q}_{4}+\mathcal{Q}_{6}\right)+2 i \mathcal{Q}_{m+n}\left(\mathcal{Q}_{1}-\mathcal{Q}_{5}\right) \\
& +2 j \mathcal{Q}_{m+n}\left(\mathcal{Q}_{2}-\mathcal{Q}_{4}\right)+4 i j \mathcal{Q}_{m+n} \mathcal{Q}_{4} \\
= & \mathcal{Q}_{m+n+3}^{\prime}-2 i \mathcal{Q}_{m+n+3}^{\prime}-j \mathcal{Q}_{m+n+3}-i j \mathcal{Q}_{m+n+3} \\
= & (1-2 i)\left(\mathcal{Q}_{m+n+3}^{\prime}-2 j \mathcal{Q}_{m+n+3}\right) .
\end{aligned}
$$

\section{Identities 2.}

$$
\begin{aligned}
& \mathcal{B}_{n+1}^{2}-\mathcal{B}_{n-1}^{2}=\mathcal{Q}_{2 n}\left[\begin{array}{ll}
3 & 2 \\
2 & 1
\end{array}\right](-1+10 i-6 j+4 i j), \\
& \mathcal{B}_{n}^{2}+\mathcal{B}_{n+1}^{2}=-\mathcal{Q}_{2 n}^{\prime}\left[\begin{array}{ll}
7 & 4 \\
4 & 3
\end{array}\right](1+10 i-2 j+4 i j) .
\end{aligned}
$$

Proof . From the equalities (2) and (5),

$$
\begin{aligned}
\mathcal{B}_{n+1}^{2}= & Q_{2 n+2}-\mathcal{Q}_{2 n+4}-\mathcal{Q}_{2 n+6}+\mathcal{Q}_{2 n+8}+2 i\left(\mathcal{Q}_{n+1} \mathcal{Q}_{n+2}-\mathcal{Q}_{n+3} \mathcal{Q}_{n+4}\right) \\
& +2 j\left(\mathcal{Q}_{n+1} \mathcal{Q}_{n+3}-\mathcal{Q}_{n+2} \mathcal{Q}_{n+4}\right)+2 i j\left(\mathcal{Q}_{n+1} Q_{n+4}+\mathcal{Q}_{n+2} \mathcal{Q}_{n+3}\right), \\
= & \mathcal{Q}_{2 n+2}-\mathcal{Q}_{2 n+4}-\mathcal{Q}_{2 n+6}+\mathcal{Q}_{2 n+8}+2 i\left(\mathcal{Q}_{2 n+3}-\mathcal{Q}_{2 n+7}\right) \\
& +2 j\left(\mathcal{Q}_{2 n+4}-\mathcal{Q}_{2 n+6}\right)+4 i j \mathcal{Q}_{2 n+5}, \\
= & -\left(\mathcal{Q}_{2 n+3}+\mathcal{Q}_{2 n+7}\right)-2 i \mathcal{Q}_{2 n+5}^{\prime}+2 j\left(\mathcal{Q}_{2 n+4}-\mathcal{Q}_{2 n+6}\right)+4 i j \mathcal{Q}_{2 n+5}, \\
= & -Q_{2 n+5}+2 i \mathcal{Q}_{2 n+5}^{\prime}-2 j \mathcal{Q}_{2 n+5}+4 i j \mathcal{Q}_{2 n+5} .
\end{aligned}
$$

Similarly, we can compute

$\mathcal{B}_{n}^{2}=-Q_{2 n+3}+2 i Q_{2 n+3}^{\prime}-2 j Q_{2 n+3}+4 i j Q_{2 n+3}$

and

$\mathcal{B}_{n-1}^{2}=-Q_{2 n+1}+2 i Q_{2 n+1}^{\prime}-2 j Q_{2 n+1}+4 i j Q_{2 n+1}$. 
Now, we have

$$
\begin{aligned}
\mathcal{B}_{n+1}^{2}-\mathcal{B}_{n-1}^{2}= & \left(-\mathcal{Q}_{2 n+5}+2 i \mathcal{Q}_{2 n+5}^{\prime}-2 j \mathcal{Q}_{2 n+5}+4 i j \mathcal{Q}_{2 n+5}\right) \\
& -\left(-\mathcal{Q}_{2 n+1}+2 i \mathcal{Q}_{2 n+1}^{\prime}-2 j \mathcal{Q}_{2 n+1}+4 i j \mathcal{Q}_{2 n+1}\right) \\
= & \mathcal{Q}_{2 n}\left[\begin{array}{ll}
3 & 2 \\
2 & 1
\end{array}\right](-1+10 i-6 j+4 i j)
\end{aligned}
$$

and

$$
\begin{aligned}
\mathcal{B}_{n}^{2}+\mathcal{B}_{n+1}^{2} & =-Q_{2 n+3}^{\prime}(1+10 i-2 j+4 i j) \\
& =-Q_{2 n}^{\prime}\left[\begin{array}{ll}
7 & 4 \\
4 & 3
\end{array}\right](1+10 i-2 j+4 i j) .
\end{aligned}
$$

Identity 3. For $n \geq 0$, The $n^{\text {th }}$ negabicomplex Fibonacci $Q_{n}$-matrix is

$$
\mathcal{B}_{-\mathrm{n}}=\frac{1}{(-1)^{n}}\left[\begin{array}{ll}
f_{n-1} \mathcal{B} f_{1}-f_{n} \mathcal{B} f_{0} & f_{n-1} \mathcal{B} f_{0}-f_{n} \mathcal{B} f_{-1} \\
f_{n-1} \mathcal{B} f_{0}-f_{n} \mathcal{B} f_{-1} & f_{n+1} \mathcal{B} f_{-1}-f_{n} \mathcal{B} f_{0}
\end{array}\right]
$$

Proof . Now, we will give proof of identity $\mathcal{B}_{-n}$. We have

$$
\begin{aligned}
\mathcal{B}_{-\mathrm{n}} & =\mathcal{Q}_{-n}+i Q_{-n+1}+j \mathcal{Q}_{-n+2}+i j \mathcal{Q}_{-n+3} \\
\mathcal{B}_{-\mathrm{n}} & =\mathcal{Q}_{-n}\left(Q_{0}+i Q_{1}+j Q_{2}+i j \mathcal{Q}_{3}\right) \\
& =\left(Q_{n}\right)^{-1}\left(Q_{0}+i Q_{1}+j Q_{2}+i j Q_{3}\right) \\
& =\left(\left[\begin{array}{cc}
f_{n+1} & f_{n} \\
f_{n} & f_{n-1}
\end{array}\right]\right)^{-1}\left[\begin{array}{cc}
1+i+2 j+3 i j & i+j+2 i j \\
i+j+2 i j & 1+j+i j
\end{array}\right] \\
& =\frac{1}{(-1)^{n}}\left[\begin{array}{cc}
f_{n-1} & -f_{n} \\
-f_{n} & f_{n+1}
\end{array}\right]\left[\begin{array}{cc}
\mathcal{B} f_{1} & \mathcal{B} f_{0} \\
\mathcal{B} f_{0} & \mathcal{B} f_{-1}
\end{array}\right] \\
& =\frac{1}{(-1)^{n}}\left[\begin{array}{cc}
f_{n-1} \mathcal{B} f_{1}-f_{n} \mathcal{B} f_{0} & f_{n-1} \mathcal{B} f_{0}-f_{n} \mathcal{B} f_{-1} \\
f_{n-1} \mathcal{B} f_{0}-f_{n} \mathcal{B} f_{-1} & f_{n+1} \mathcal{B} f_{-1}-f_{n} \mathcal{B} f_{0}
\end{array}\right],
\end{aligned}
$$

where $\mathcal{B} f_{1}, \mathcal{B} f_{0}$ and $\mathcal{B} f_{-1}$ are bicomplex Fibonacci numbers.

\section{Some Applications On Bicomplex Fibonacci $Q$-Matrix}

Let $\mathcal{B}_{n}$ be the $n^{\text {th }}$ bicomplex Fibonacci $Q_{n}$-matrix, for $n \geq 0$, these number is $2^{\text {th }}$ linear recurrence sequence. Then, we suppose the sets of $\mathbb{C}_{2}$ and $\mathbb{C}_{2}^{\prime}$ are

$\mathbb{C}_{2}=\left\{\mathcal{B}_{n} \mid \mathcal{B}_{n}=Q_{n}+i Q_{n+1}+j Q_{n+2}+i j \mathcal{Q}_{n+3}, \mathcal{Q}_{n}\right.$ is $n^{\text {th }} \mathcal{Q}$-matrix $\}$,

and

$\mathbb{C}_{2}^{\prime}=\left\{\mathcal{B}_{n} \mid \mathcal{B}_{n}=\left[\begin{array}{ll}\alpha_{n} & \beta_{n} \\ \beta_{n} & \alpha_{n}\end{array}\right] ; \alpha_{n}, \beta_{n} \in \mathbb{C}\right\}$

Then, there is an isomorphism between $\mathbb{C}_{2}$ and $\mathbb{C}_{2}^{\prime}$, in that case, we can write 
$\mathcal{B}_{\mathrm{n}}=\left(Q_{n}, Q_{n+1}, Q_{n+2}, Q_{n+3}\right) \rightarrow \mathcal{B}_{n}=\left[\begin{array}{cc}\mathcal{Q}_{n}+i Q_{n+1} & \mathcal{Q}_{n+2}+i Q_{n+3} \\ \mathcal{Q}_{n+2}+i Q_{n+3} & \mathcal{Q}_{n}+i Q_{n+1}\end{array}\right]$

Thus, we can write

$\mathbb{C}_{2}^{\prime}=\left\{\mathcal{B}_{\mathrm{n}} \mid \mathcal{B}_{\mathrm{n}}=\alpha_{\mathrm{n}}\left[\begin{array}{ll}1 & 0 \\ 0 & 1\end{array}\right]+\beta_{\mathrm{n}}\left[\begin{array}{ll}0 & 1 \\ 1 & 0\end{array}\right] ; \alpha_{n}, \beta_{\mathrm{n}}\right.$ is $n^{\text {th }}$ complex Fibonacci $\mathcal{Q}$-matrix $\}$

and

$\mathcal{B}_{\mathrm{n}}=\mathcal{Q}_{n} \mathrm{U}_{1}+\mathcal{Q}_{n+1} \mathrm{U}_{2}+\mathcal{Q}_{n+2} \mathrm{U}_{3}+\mathcal{Q}_{n+3} \mathrm{U}_{4}$

where

$\mathcal{B}_{n}=Q_{n}\left[\begin{array}{ll}1 & 0 \\ 0 & 1\end{array}\right]+Q_{n+1}\left[\begin{array}{ll}i & 0 \\ 0 & i\end{array}\right]+\mathcal{Q}_{n+2}\left[\begin{array}{ll}0 & j \\ j & 0\end{array}\right]+Q_{n+3}\left[\begin{array}{cc}0 & i j \\ i j & 0\end{array}\right]$

Since $\operatorname{det} \mathcal{B}_{\mathrm{n}} \neq 0$, there is the inverse of matrix $\mathcal{B}_{\mathrm{n}}$ and it is in $\mathbb{C}_{2}^{\prime}$. Now, let's define the $n^{\text {th }}$ bicomplex Fibonacci $\mathcal{Q}$-vector $\overrightarrow{\mathcal{B}}_{\mathrm{n}}$ and the $n^{\text {th }}$ bicomplex Lucas $\mathcal{Q}^{\prime}$-vector $\overrightarrow{\mathcal{B}}_{\mathrm{n}}^{\prime}$ as

$\overrightarrow{\mathcal{B}}_{\mathrm{n}}=i Q_{n+1}+j Q_{n+2}+i j Q_{n+3}$ and $\overrightarrow{\mathcal{B}}_{\mathrm{n}}^{\prime}=i Q_{n+1}^{\prime}+j Q_{n+2}^{\prime}+i j Q_{n+3}^{\prime}$, respectively.

Theorem 1. Let $\overrightarrow{\mathcal{B}}_{\mathrm{n}}$ and $\overrightarrow{\mathcal{B}}_{\mathrm{n}+1}$ be bicomplex Fibonacci $\mathcal{Q}$-vectors. The dot and cross product of these vectors are defined by

$<\overrightarrow{\mathcal{B}}_{\mathrm{n}}, \overrightarrow{\mathcal{B}}_{\mathrm{n}+1}>=\mathcal{Q}_{n+1} \mathcal{Q}_{n+2}+\mathcal{Q}_{n+2} \mathcal{Q}_{n+3}+\mathcal{Q}_{n+3} \mathcal{Q}_{n+4}$

and

$\overrightarrow{\mathcal{B}}_{\mathrm{n}} \times \overrightarrow{\mathcal{B}}_{\mathrm{n}+1}=\operatorname{det}\left[\begin{array}{ccc}-i & j & i j \\ \mathcal{Q}_{n+1} & \mathcal{Q}_{n+2} & \mathcal{Q}_{n+3} \\ \mathcal{Q}_{n+2} & \mathcal{Q}_{n+3} & \mathcal{Q}_{n+4}\end{array}\right]$

where in the permanent of $\overrightarrow{\mathcal{B}}_{\mathrm{n}} \times \overrightarrow{\mathcal{B}}_{\mathrm{n}+1}$, the signatures of the permutations are not taken into account, [16].

Proof : From the equality (2), we obtain,

$$
\begin{aligned}
<\overrightarrow{\mathcal{B}}_{\mathrm{n}}, \overrightarrow{\mathcal{B}}_{\mathrm{n}+1}> & =\mathcal{Q}_{n+1} \mathcal{Q}_{n+2}+\mathcal{Q}_{n+2} \mathcal{Q}_{n+3}+\mathcal{Q}_{n+3} \mathcal{Q}_{n+4} \\
& =\mathcal{Q}_{2 n+3}+\mathcal{Q}_{2 n+5}+\mathcal{Q}_{2 n+7} \\
& =\mathcal{Q}_{2 n}\left(\mathcal{Q}_{3}+\mathcal{Q}_{5}+\mathcal{Q}_{7}\right) \\
& =\left[\begin{array}{cc}
f_{2 n+1} & f_{2 n} \\
f_{2 n} & f_{2 n-1}
\end{array}\right]\left[\begin{array}{ll}
f_{4}+f_{6}+f_{8} & f_{3}+f_{5}+f_{7} \\
f_{3}+f_{5}+f_{7} & f_{2}+f_{4}+f_{6}
\end{array}\right] \\
& =4 \mathcal{Q}_{2 n}\left[\begin{array}{cc}
8 & 5 \\
5 & 3
\end{array}\right] .
\end{aligned}
$$

Now, we will calculate $\overrightarrow{\mathcal{B}}_{n} \times \overrightarrow{\mathcal{B}}_{n+1}$,

$=-i\left(Q_{n+2} Q_{n+4}+Q_{n+3} Q_{n+3}\right)-j\left(Q_{n+1} Q_{n+4}+Q_{n+2} Q_{n+3}\right)+i j\left(Q_{n+1} Q_{n+3}+Q_{n+2} Q_{n+2}\right)$ 
$=2\left(-i Q_{2 n+6}-j Q_{2 n+5}+i j Q_{2 n+4}\right)$

$=2\left(i Q_{2 n+2}+j Q_{2 n+3}+i j Q_{2 n+4}\right)-2 i\left(Q_{2 n+6}+Q_{2 n+2}\right)-2 j\left(Q_{2 n+5}+Q_{2 n+3}\right)$,

finally, we have

$=2\left(\overrightarrow{\mathcal{B}}_{2 n+1}-3 i Q_{2 n+4}-j Q_{2 n+4}^{\prime}\right)$

where $\overrightarrow{\mathcal{B}}_{2 n+1}$ is bicomplex Fibonacci vector, $Q_{2 n+4}$ and $Q_{2 n+4}^{\prime}$ are Fibonacci $Q$-matrix and Lucas $\mathcal{Q}^{\prime}$-matrix, respectively.

Example 1. Let $\overrightarrow{\mathcal{B}}_{1}$ and $\overrightarrow{\mathcal{B}}_{2}$ be bicomplex Fibonacci $\mathcal{Q}$-vectors such that $\overrightarrow{\mathcal{B}}_{1}=i \mathcal{Q}_{2}+j \mathcal{Q}_{3}+i j \mathcal{Q}_{4}$ and $\overrightarrow{\mathcal{B}}_{2}=i Q_{3}+j \mathcal{Q}_{4}+i j \mathcal{Q}_{5}$. The dot product of these vectors are

$<\overrightarrow{\mathcal{B}}_{1}, \overrightarrow{\mathcal{B}}_{2}>=\mathcal{Q}_{2} \mathcal{Q}_{3}+\mathcal{Q}_{3} \mathcal{Q}_{4}+\mathcal{Q}_{4} \mathcal{Q}_{5}=\mathcal{Q}_{5}+\mathcal{Q}_{7}+\mathcal{Q}_{9}$

from the equality (2) and the equalities of Fibonacci numbers in [3, 5], we obtain

$=3 Q_{7}+Q_{7}=4 Q_{7}$

$=4\left[\begin{array}{cc}21 & 13 \\ 13 & 8\end{array}\right]$

\section{Acknowledgment}

Thank you to the referee for the careful reading, suggestion and valuable comments in this article.

\section{Conclusion}

In this present work we have given, firstly we described the bicomplex numbers with coefficients from the Fibonacci $Q$-matrix and Lucas $Q^{\prime}$-matrix sequences. We have given many equations that hold an important place in the literature on these numbers. For further studies, we plan to describe some additional identities and properties for these new numbers.

\section{Statement of Research and Publication Ethics}

The author declares that this study complies with Research and Publication Ethics

\section{References}

[1] Price G. B. 1991. An Introduction to Multicomplex Spaces and Functions, Marcel Dekker.Inc.New York, I(1)-44(1).

[2] Nurkan S. K., Güven I. A. 2018. A note on bicomplex Fibonacci and Lucas numbers. International Journal of Pure and Applied Mathematics, 120 (3): 365-377. doi 10.12732/ijpam.v120i3.7.

[3] Dunlap R. A. 1997. The Golden Ratio and Fibonacci Numbers. World Scientific Press.

[4] Vajda S. 1989. Fibonacci and Lucas numbers, and the Golden Section. Theory and Applications, Halsted Press.

[5] Hoggatt V. E. 1969. Fibonacci and Lucas Numbers published by The Fibonacci Association.

[6] Iyer M. R. 1969. Some results on Fibonacci quaternions. The Fibonacci Quarterly, 7 (2): 201210.

[7] Knuth D. 2013. Negafibonacci Numbers and Hyperbolic Plane, Annual Meeting of the Math. Association of America.

[8] Horadam A. F. 191. A Generalized Fibonacci Sequence. American Math. Monthly, 68. 
[9] Iyer M. R. 1969. Identities involving generalized Fibonacci numbers. The Fibonacci Quarterly, 7 (1): 66-72.

[10] Lucas E. 1961. Théorie des Numbers. Nf. Balanchard, Paris, 1 (520): 25.

[11] Koshy T. 2001. Fibonacci and Lucas Numbers with Applications. Wiley-Intersience Publication, New York.

[12] Basin S. L., Hoggatt V. E. 1963. A primer on the Fibonacci sequence, Part II. Fib. Quart., 1: 6168.

[13] Brenner J. L. 1951. June Meeting of the Pacific Northwest Section. 1. Lucas Matrix. Amer. Math. Monthly, 58: 220-221.

[14] Honsberger R. 1985. The Matrix Q. Mathematical Gems III. Washington, DC: Math. Assoc. Amer., 106-107.

[15] King C. H. 1960. Some Further Properties of the Fibonacci Numbers. Master's thesis. San Jose, CA: San Jose State.

[16] Glynn D. G. 2010. Permanenet of a square matrix. European Journal of Combinatorics, 31 (7): 1891-1897. 